\title{
Nature of Interactions at the Interface of Two Water-Saturated Commercial $\mathrm{TiO}_{2}$ Polymorphs
}

\author{
Pellegrino Conte, ${ }^{*}, \dagger, \S$ Vittorio Loddo, ${ }^{\ddagger}, \|$ Claudio De Pasquale, ${ }^{\dagger, \S}$ Valentina Marsala, ${ }^{\dagger, \S}$
}

Giuseppe Alonzo, ${ }^{\dagger} \S$ and Leonardo Palmisano ${ }^{\ddagger} \|$

${ }^{\dagger}$ Dipartimento di Scienze Agrarie e Forestali, Università degli Studi di Palermo, v.le delle Scienze, Edificio 4, 90128, Palermo, Italy

${ }^{\ddagger}$ Dipartimento di Energia, Ingegneria dell'Informazione, e Modelli Matematici (DEIM), Università degli Studi di Palermo, v.le delle Scienze, Edificio 6, 90128 - Palermo, Italy

${ }^{\S}$ Consorzio Interuniversitario Nazionale Chimica e Tecnologie per l'Ambiente (INCA) UdR Palermo 2

${ }$ Consorzio Interuniversitario Nazionale Chimica e Tecnologie per l'Ambiente (INCA) UdR Palermo 1

Supporting Information

ABSTRACT: Two commercial $\mathrm{TiO}_{2}$ samples, a $100 \%$ anatase and a $100 \%$ rutile, were used for the fast field cycling NMR experiments. The results showed a different behavior between the different samples. In particular, water molecules were unbonded to the solid surface for the rutile sample, whereas they appeared to chemically interact with the surface through H-bond formation with the anatase sample. The above findings accord with the generally lower activity of rutile with respect to anatase reported in literature for photocatalytic oxidation reactions in water. The difficulty of water to interact with rutile surface, indeed, could hinder the formation of $\mathrm{OH}$ radicals, which are the most important oxidant species.

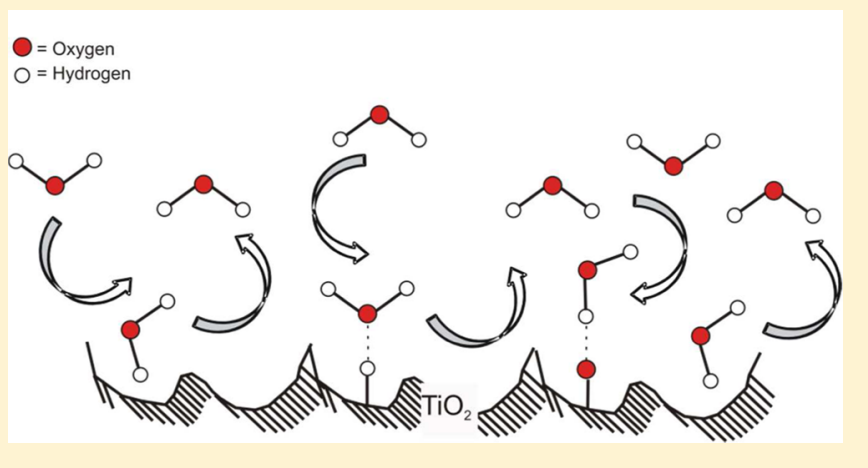

O= Oxygen $\mathrm{O}=$ Hydrogen

\section{INTRODUCTION}

Titanium dioxide either powdered or in the form of thin films supported on different kinds of materials is widely applied in photocatalysis for pollutant abatement, ${ }^{1-5}$ partial oxidation, or reduction of organic and inorganic molecules in chemical syntheses, ${ }^{6-8}$ in solar cells for the production of hydrogen, as a gas sensor, as a white pigment such as in paints and cosmetics, as an optical coating, as a corrosion-protective coating, and in many electric devices. 9 ' Titanium dioxide also plays a very important role in biocompatibility for bone implants and in the realization of electronic components such as those applied for nuclear magnetic resonance instruments. ${ }^{9}$

All peculiarities of titanium dioxide depend on the surface characteristics of such material, ${ }^{10,11}$ which, in turn, are affected by the preparation methods applied for its achievement. ${ }^{5,12}$ In fact, it is well-recognized that polymorphism, crystallinity degree, and amount of surface defects are all dependent on the nature of the parent material and the temperature and pressure achieved during $\mathrm{TiO}_{2}$ preparation. ${ }^{1,4,5,9,12,13}$

Because of its properties, titanium dioxide is widely applied in green chemistry, where water is used as a "green" solvent due to its null or negligible environmental impact as compared with the traditional solvents employed in organic and inorganic syntheses. ${ }^{14}$ For this reason, studies concerning the way how water binds to $\mathrm{TiO}_{2}$ surface are of paramount importance. ${ }^{9,14}$ In fact, as an example, $\mathrm{TiO}_{2}$ selectivity for organic molecules appears to be affected by wet adsorption conditions, ${ }^{13,15}$ whereas the efficaciousness of the redox transformations on titanium dioxide catalysts strongly depends on the way how water molecules dissociate on the $\mathrm{TiO}_{2}$ solid surface. ${ }^{9}$

Many papers, mostly reviewed in Henderson ${ }^{14}$ and Diebold, ${ }^{9}$ deal with the interactions of water on the surface of pure crystalline $\mathrm{TiO}_{2}$ polymorphs. The majority of such papers concern mainly theoretical evaluation of water- $\mathrm{TiO}_{2}$ interactions. Only a few results were experimentally retrieved primarily on the crystalline forms of rutile $\mathrm{TiO}_{2}$ (indicated simply as rutile from now-on) by applying, as an example, ultraviolet photoemission spectroscopy, electron energy loss spectroscopy, Auger electron spectroscopy, X-ray photoelectron spectroscopy, and temperature-programmed desorption investigations. ${ }^{9}$ The interactions between water and anatase $\mathrm{TiO}_{2}$ (that will be further indicated only as anatase) have been, up to now, investigated only theoretically. ${ }^{9}$ Just a few X-ray photoelectron spectroscopy experiments have been conducted on water-anatase interactions due to the lower importance of such a polymorph as compared with rutile. ${ }^{9}$ To the best of our knowledge, the interaction of water with the surface of the commercial forms of rutile and anatase has not yet been investigated. This investigation is very important due to the prevalence of such materials on the market and to

Received: January 10, 2013

Revised: February 14, 2013

Published: February 15, 2013 
explain the reason why commercial rutile appears less effective than commercial anatase. ${ }^{10,11}$ The aim of the present article is, then, the evaluation of the nature of the interactions between water and commercial rutile and anatase by using fast field cycling (FFC) NMR relaxometry whose peculiarities are reported in the Supporting Information.

\section{MATERIALS AND METHODS}

Samples. Rutile and anatase were purchased by SigmaAldrich (Milan, Italy) and Merck (Milan, Italy), respectively. Their surface area (SA) was measured by the dynamic Brunauer-Emmett-Teller (BET) method using a Micromeritics Flowsorb 2309 apparatus (Dunstable, U.K.) with nitrogen as the adsorbate. SA of rutile was $2.5 \mathrm{~m}^{2} \cdot \mathrm{g}^{-1}$, whereas that of anatase was $10 \mathrm{~m}^{2} \cdot \mathrm{g}^{-1}$.

Fast Field Cycling NMR Experiments. $552.0 \mathrm{mg}$ of rutile and $512.9 \mathrm{mg}$ of anatase were suspended in 3.0206 and 3.0081 $\mathrm{g}$ of deionized water, respectively. The suspensions were allowed to sediment overnight prior to the relaxometry investigations. The samples were put in the probe of a Stelar Smartracer fast-field-cycling relaxometer (Stelar s.r.l., Mede, PV-Italy) and analyzed at 298, 313, 333, 343, and $353 \mathrm{~K}$.

The basic theory about FFC NMR relaxometry is reported in Kimmich and Anoardo ${ }^{16}$ and Ferrante and Sykora. ${ }^{17}$ The details of the FFC NMR sequence used in the present study have already been published. ${ }^{18}$ They are also reported in the Supporting Information together with the experimental details that have already been reported in Conte et al. ${ }^{19}$ The only differences between the parameters used in the present study and those reported in Conte et al. ${ }^{19}$ concern the proton Larmor frequencies (PLFs) used to perform the analyses. Here the PLFs of the relaxing magnetic field $\left(B_{\mathrm{RLX}}\right)$ were varied from 0.01 up to $10 \mathrm{MHz}$, the proton Larmor frequency of the polarization field $\left(B_{\mathrm{POL}}\right)$ for the prepolarized sequence (De Pasquale et al., 2012) was set at $10 \mathrm{MHz}$, whereas the PLF for free induction decay acquisition $\left(B_{\mathrm{ACQ}}\right.$, see Supporting Information) was set at $7.2 \mathrm{MHz}$. The nuclear magnetic resonance dispersion (NMRD) curves, obtained by measuring the longitudinal relaxation rates $\left(R_{1}=1 / T_{1}\right)$ at each $B_{\mathrm{RLX}}$ (see Supporting Information), provide information about the spectrum of the reorientational and diffusional molecular dynamics. ${ }^{16}$ All measurements were repeated twice. The error on each $R_{1}$ value was $10 \%$. Figure 1 reports the relaxation rates versus PLFs (i.e., NMRD profiles) at each temperature with error bars on $R_{1}$.

\section{RESULTS}

Figure 1 reports the NMRD profiles of rutile and anatase $\mathrm{TiO}_{2}$ polymorphs at different temperatures. All profiles showed the classical stretched Lorentzian shapes. ${ }^{16}$ In particular, the profiles retrieved for rutile were at slower $R_{1}$ values (Figure 1A) than those acquired for anatase (Figure 1B).

The temperature dependence of the NMRD profiles for rutile and anatase revealed opposite trends. In fact, whereas rutile profiles showed $R_{1}$ values decreasing with temperature enhancement (Figure 1A), anatase profiles revealed a direct proportionality between proton longitudinal relaxation rate values and temperature (Figure 1B). In addition, at temperatures greater than $343 \mathrm{~K}$, the NMRD profiles of anatase appeared to cross those obtained at lower temperatures (from 298 to $333 \mathrm{~K}$ ) for the same water-saturated material as the PLF became lower than $0.1 \mathrm{MHz}$ (Figure 1B). $(\alpha)$

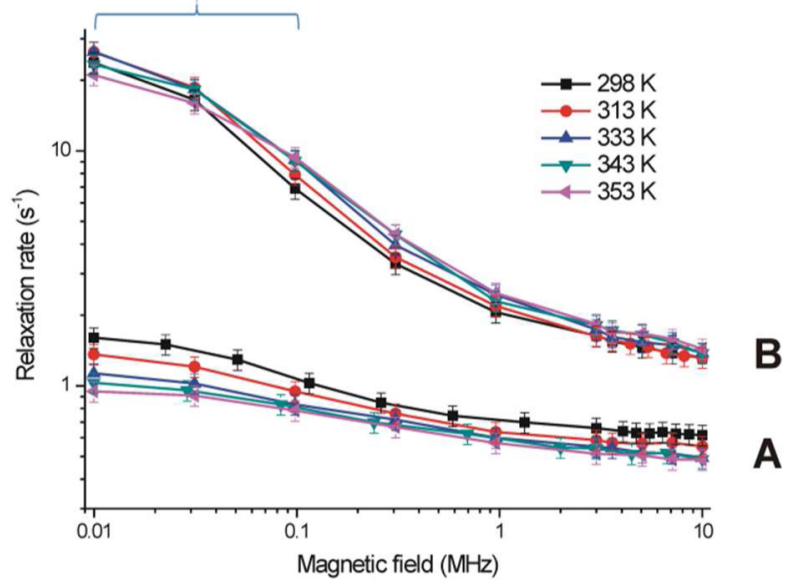

Figure 1. NMRD profiles at different temperatures of rutile (A) and anatase (B). $(\alpha)$ is the proton Larmor frequency region of the applied magnetic field where the NMRD profiles of anatase at 343 and $353 \mathrm{~K}$ cross those at the lowest temperatures (298, 313, and $333 \mathrm{~K})$.

\section{DISCUSSION}

Water dynamics on the surface of porous media is related to SA values. The latter, in turn, depends on pore sizes. ${ }^{20}$ In fact, the smaller the pore sizes, the larger the SA value. Conversely, SA reduction is achieved when the sizes of the pores increase. As water molecules flow through larger sized pores, their motion occurs at a frequency that is broader than that of water molecules constrained in smaller sized pores. For this reason, quickly moving water cannot efficaciously interact with either the neighboring molecules or with the molecular sites on the surface at the liquid-solid interface. As a consequence, intermolecular dipolar interactions are weakened and a reduction of the proton longitudinal relaxation rate (shorter $R_{1}$ values) can be observed compared with the $R_{1}$ values for slowly moving or immobilized water systems. ${ }^{21}$

In the present study, SAs of rutile and anatase resulted 2.5 and $10 \mathrm{~m}^{2} \cdot \mathrm{g}^{-1}$, respectively. It is then expected that $R_{1(\text { rutile })}<$ $R_{1 \text { (anatase) }}$. Figure 1 confirms this expectation. In fact, all NMRD profiles achieved for the water saturated rutile are placed at shorter $R_{1}$ values (Figure $1 \mathrm{~A}$ ) compared with the profiles of the water-saturated anatase (Figure 1B).

The feasibility of the proposed water molecular mobility mechanism was also investigated by variable temperature experiments. In fact, it is well-recognized that the time spent by water on the surface of porous media decreases as temperature is increased. ${ }^{16}$ As water mobility is increased because of temperature enhancement, dipolar interactions at the solid-liquid interface are weakened. This leads to a longer time for proton relaxation (shorter $R_{1}$ values). ${ }^{21} \mathrm{~A}$ close look at the temperature dependence of the NMR profiles in Figure 1 reveals that the aforementioned expectation (i.e., NMRD profiles moving toward shorter $R_{1}$ values with temperature enhancement) was achieved only for the water molecules saturating rutile (Figure 1A). Conversely, water-saturating anatase provided NMRD profiles that were not only translated toward longer $R_{1}$ values but also changed in slope. In fact, the NMRD curves obtained at 343 and $353 \mathrm{~K}$ crossed those retrieved at the lowest temperatures (Figure 1B). Clearly, pore size cannot be the only factor affecting water dynamics on the surface of the two titanium dioxide polymorphs. 
Lauffer, ${ }^{22}$ Korb, $^{23}$ Kimmich and Anoardo, ${ }^{16}$ Korb and Bryant, $^{24}$ and Laurent et al. ${ }^{25}$ reported that the correct chemical-physical approach to understand the molecular dynamics of a fluid on a solid surface must be based on two possible mechanisms. From the one side, water diffuses by the porous medium surface, from a distance " $d$ ", usually indicated as distance of closest approach, and infinity. ${ }^{26}$ This diffusion occurs when nonchemically interacting water is replaced by another similar molecule belonging to the bulk water system (Figure 2A). The time spent by water on the solid surface can

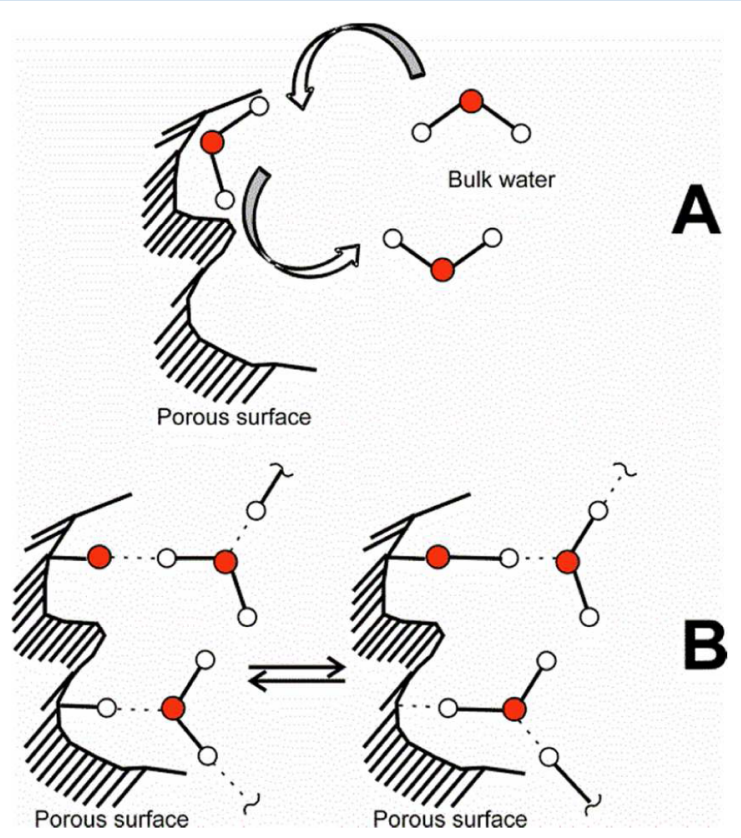

Figure 2. Mechanisms of water dynamics on porous media surfaces. Water diffusion can be dependent only on pore sizes (A) or it can be mediated by hydrogen bonds, which, in turn, are subjected to rapid exchanges (B). The dashed lines are the H-bonds; the red dots are the oxygen atoms; the white dots represent the hydrogen atoms.

be affected by the formation of $\mathrm{H}$ bonds due to the presence of either hydrogen acceptors or hydrogen donors on the outer boundary. ${ }^{19}$ Following H-bond formation, hydrogen atoms can also be subjected to rapid exchanges between water molecules and the porous surfaces, as depicted in Figure 2B. The mathematics of the aforementioned mechanism is described by eq $1::^{22,25}$

$$
R_{1}=Y \frac{[\mathrm{C}]}{d D}\left[7 J\left(\omega_{\mathrm{S}} \tau_{\mathrm{D}}\right)+3 J\left(\omega_{\mathrm{I}} \tau_{\mathrm{D}}\right)\right]+\frac{f_{\mathrm{M}}}{T_{1 \mathrm{M}}+\tau_{\mathrm{M}}}
$$

Here [C] is the amount of paramagnetic centers, $d$ is the distance of the closest approach, $D$ is the diffusion coefficient, and $Y$ is a constant. The latter accounts for the Avogadro's number $\left(N_{\mathrm{A}}\right)$, the Plank constant, the quantum spin number and the magnetogyric ratios $(\gamma)$ of the observed nuclei $\left({ }^{1} \mathrm{H}\right)$, and the paramagnetic centers. $J\left(\omega_{\mathrm{i}} \tau_{\mathrm{D}}\right)$ is the spectral density depending on the Larmor frequency of the electrons in paramagnetic centers $(S)$ and that of proton nuclei (I). $f_{M}$ represents the molar fraction of water chemically bound to the porous system (BW), $T_{1 \mathrm{M}}$ is the proton longitudinal relaxation time of the chemically bound water, and $\tau_{\mathrm{M}}$ is the exchange correlation time, which measures the mean residence time of the bound water. ${ }^{19}$
The first term on the right side of eq 1 describes the diffusional motion of water as depicted in Figure 2A, whereas the second term represents the water motion mediated by hydrogen bonds, as represented in Figure 2B. Because of the absence of paramagnetic centers in the two $\mathrm{TiO}_{2}$ polymorphs used for the present study, the contribution of the first term in eq 1 can be neglected. For this reason, the longitudinal relaxation rate of the water saturated $\mathrm{TiO}_{2}$ polymorphs depends on $\mathrm{BW}$ amount, on $\mathrm{BW}$ proton longitudinal relaxation time, and on the exchange correlation time.

Two different cases must be considered. When $\tau_{\mathrm{M}} \ll T_{1 \mathrm{M}}$ fast motion regime occurs. ${ }^{19}$ Under this condition, the proton longitudinal relaxation rate is proportional to $1 / T_{1 \mathrm{M}}$. In particular, the proton exchange between water and $\mathrm{TiO}_{2}$ surface occurs, as depicted in Figure 2A, thereby allowing the consideration that water is not chemically retained on the titanium dioxide surface. According to Lauffer, ${ }^{22}$ the proton longitudinal relaxation time value of the unbound water decreases as temperature is increased. In fact, $T_{1 \mathrm{M}}$ contains a dipolar (i.e., through space) and a scalar, or contact (i.e., through bonding-electrons), relaxation contribution. ${ }^{22}$ Both of them reveal a reduced efficiency as temperature is increased. For this reason, reduction of $R_{1}$ values in the whole range spanned by FFC NMR relaxometry can be measured. ${ }^{21}$

As $\tau_{\mathrm{M}} \gg T_{1 \mathrm{M}}$, slow motion regime occurs. ${ }^{19}$ In this case, the proton longitudinal relaxation rate depends upon $1 / \tau_{\mathrm{M}}$ and water appears bound to the porous medium surface due to the formation of $\mathrm{H}$ bonds (Figure 2B). Because of the inverse relationship between correlation time and temperature, ${ }^{21}$ an increase in $R_{1}$ values is achieved as temperature is enhanced.

On the basis of the mechanism founded on eq 1 , we can conclude that water molecules interact chemically to the surface of anatase through $\mathrm{H}$-bond formation. Conversely, water is virtually unbound to the surface of rutile.

It is worth noting that the lack of chemical interactions between water and rutile found by FFC NMR relaxometry accords with its generally low photochemical activity. ${ }^{6,10,27}$ In fact, low photoactivity of commercial rutile has been observed when it is used for both photomineralization and selective photooxidation reactions in water. 6,10

The mechanism of the photocatalytic oxidation reactions foresees the formation of $\cdot \mathrm{OH}$ radicals that are believed to be responsible for the primary oxidant attack to the substrate. The interaction between adsorbed water and $\mathrm{TiO}_{2}$ surface plays the major role to produce the oxidant species according to the steps reported in Scheme 1. Consequently, the absence of chemical

Scheme 1. Mechanisms of Photo-Oxidation Reactions Occurring on the $\mathrm{TiO}_{2}$ Surface

$$
\begin{aligned}
& \mathrm{TiO}_{2} \stackrel{\mathrm{hv}}{\longrightarrow} \mathrm{TiO}_{2}\left(\mathrm{e}_{\mathrm{CB}}^{-}, \mathrm{h}_{\mathrm{VB}}^{+}\right) \\
& \mathrm{H}_{2} \mathrm{O}+\mathrm{h}_{\mathrm{VB}}^{+} \stackrel{\mathrm{TiO}_{2}}{\longrightarrow} \cdot \mathrm{OH}+\mathrm{H}^{+} \\
& \cdot \mathrm{OH}+\text { substrate } \rightarrow \text { oxidised products } \\
& \text { oxidised products } \stackrel{\mathrm{OH}}{\longrightarrow} \stackrel{\mathrm{OH}}{\longrightarrow} \mathrm{H}_{2} \mathrm{O}+\mathrm{CO}_{2}+\text { inorganic species }
\end{aligned}
$$

interactions between $\mathrm{H}_{2} \mathrm{O}$ and the surface of rutile, as highlighted by NMRD results, strongly suggests that the formation of $\cdot \mathrm{OH}$ radicals from $\mathrm{H}_{2} \mathrm{O}$, according to eq 3 in Scheme 1, is highly improbable. However, it must be stated that high levels of rutile photoactivity, for both photomineralization and selective photo-oxidation reactions in water, can be 
attained when this catalyst is prepared under milder experimental conditions. ${ }^{10,28}$

Although our results accord with some literature data, $6,10,27$ they appear to discord with those provided in Beck et al., ${ }^{29}$ Jug et al., ${ }^{30}$ and Erdogan et al. ${ }^{31}$ In fact, those authors reported that water can interact with rutile surface mainly in its dissociate form (hydroxylated rutile). The discrepancy between the NMRD results reported here and those aforementioned can be accounted for in two different ways. From the one hand, Beck et al. $^{29}$ used two different rutile materials in their experiments. One of the rutile forms showed a SA of $11 \mathrm{~m}^{2} \cdot \mathrm{g}^{-1}$, whereas the second had a SA of $30 \mathrm{~m}^{2} \cdot \mathrm{g}^{-1}$. As reported in the Materials and Methods, we used a rutile having a SA of 2.5 $\mathrm{m}^{2} \cdot \mathrm{g}^{-1}$. It is known that the amount of hydroxylated $\mathrm{TiO}_{2}$ sites increases as SA becomes larger. ${ }^{10}$ For this reason, the number of $\mathrm{H}$ bonds that water can form with rutile is directly proportional to the SA value. Because of the low number of $\mathrm{H}$ bonds conceivably present in the rutile used in the present study, it is possible to argue that FFC NMR relaxometry was unable to reveal the interactions between water and the rutile surface. Jug et al. ${ }^{30}$ and Erdogan et al. ${ }^{31}$ reported about computational calculations on cyclic cluster models of rutile pure crystals to achieve information about the energy involved in the different ways water can interact with rutile. For this reason, a comparison between our results and those described in Jug et al. ${ }^{30}$ and Erdogan et al. ${ }^{31}$ is not feasible.

At the largest temperature values ( 343 and $353 \mathrm{~K}$ ), the NMRD profiles of anatase crossed those retrieved at temperatures in the range of $298-333 \mathrm{~K}$ as the PLF of the applied magnetic field became lower than $0.1 \mathrm{MHz}$ (Figure 1B). A possible explanation of such a behavior is related to the interruption of the diffusive processes at the solid-liquid interface by the chemical exchanges with the bulk water. ${ }^{23}$

The findings reported on anatase in the present study appear to confirm the theoretical calculations provided by Vittadini et al. $^{32}$ In fact, these authors reported that water is molecularly adsorbed on the surface of anatase through the formation of hydrogen bondings. In addition, they also reported that temperature enhancement favors water dissociation and hydroxylation of anatase surface. However, we do think that the anomalous behavior at high temperatures reported in Figure 1B cannot be attributed to water dissociation and surface hydroxylation. As suggested by Korb, ${ }^{23}$ it can be due to the alteration of the diffusive processes as a consequence of the chemical exchanges becoming important as $T \geq 343 \mathrm{~K}$. The temperature needed for water dissociation on the $\mathrm{TiO}_{2}$ surface must be at least $>400 \mathrm{~K}^{29}$

\section{CONCLUSIONS}

The present article shows for the first time the nature of the interactions between water and the surface of two commercially available titanium dioxide polymorphs. In particular, a low SA rutile produced by Sigma-Aldrich was compared with a high SA anatase produced by Merck. Results indicate that the low specific SA of rutile prevented the formation of chemical interactions with water. The above findings justify the low photoactivity observed when rutile samples, prepared at high temperatures such as the Sigma-Aldrich one, are used for both photomineralization and selective photooxidation reactions in water. Conversely, hydrogen bonds between water and the surface of anatase sample have been hypothesized to explain the slow motion regime revealed by FFC NMR relaxometry experiments. The presence of $\mathrm{H}$ bonds not only explained the better anatase photoreactivity as compared with the rutile above but also confirmed literature results that have been only theoretically obtained.

To the best of our knowledge this is the first time an NMR technique has been applied to distinguish the different surface properties of commercially available $\mathrm{TiO}_{2}$ polymorphs. The potentiality showed by FFC NMR relaxometry in revealing differences between rutile and anatase will be further applied to investigate the effects of SAs and crystallinity degree on water motion regimes.

\section{ASSOCIATED CONTENT}

\section{Supporting Information}

The bases for FFC NMR relaxometry and the details of the experimental conditions for relaxometry. This material is available free of charge via the Internet at http://pubs.acs.org

\section{AUTHOR INFORMATION}

\section{Corresponding Author}

*E-mail: pellegrino.conte@unipa.it. Tel: 003909123864673. Fax: 0039091484035.

\section{Author Contributions}

The manuscript was written through contributions of P.C., L.P., and V.L. All authors have given approval to the final version of the manuscript. All the authors contributed equally to the experiments.

\section{Notes}

The authors declare no competing financial interest.

\section{REFERENCES}

(1) Schiavello, M. Photocatalysis and Environment Trends and Applications; Kluwer Academic: Dordrecht, The Netherlands, 1988.

(2) Serpone, N.; Pelizzetti, E. Photocatalysis, Fundamentals and Applications; J. Wiley \& Sons: Chichester, U.K., 1988.

(3) Ollis, D. F.; El-Ekabi, H. Photocatalytic Purification and Treatment of Water and Air; Elsevier Science: New York, 1993.

(4) Schiavello, M. Heterogeneous Photocatalysis; Wiley Series in Photoscience and Photoengineering 3; J. Wiley \& Sons: Chichester, U.K., 1997.

(5) Augugliaro, V.; Loddo, V.; Pagliaro, M.; Palmisano, G.; Palmisano, L. Clean by Light Irradiation: Practical Application of Supported $\mathrm{TiO}_{2}$; RCS Publishing: Cambridge, U.K., 2010.

(6) Palmisano, G.; Yurdakal, S.; Augugliaro, V.; Loddo, V.; Palmisano, L. Adv. Synth. Catal. 2007, 349, 964.

(7) Palmisano, G.; García-López, E.; Marcì, G.; Loddo, V.; Yur-dakal, S.; Augugliaro, V.; Palmisano, L. Chem. Commun. 2010, 46, 7074.

(8) Augugliaro, V.; Bellardita, M.; Loddo, V.; Palmisano, G.; Palmisano, L.; Yurdakal, S. J. Photochem. Photobiol. 2012, C, 224.

(9) Diebold, U. Surf. Sci. Rep. 2003, 48, 53.

(10) Sclafani, A.; Palmisano, L.; Schiavello, M. J. Phys. Chem. 1990, 94, 829.

(11) Sclafani, A.; Palmisano, L.; Davì, E. New J. Chem. 1990, 14, 265.

(12) Augugliaro, V.; Caronna, T.; Loddo, V.; Marcì, G.; Palmisano, G.; Palmisano, L.; Yurdakal, S. Chem. Eur. J. 2008, 14, 4640.

(13) Yurdakal, S.; Palmisano, G.; Loddo, V.; Alagöz, O.; Augugliaro, V.; Palmisano, L. Green Chem. 2009, 11, 510.

(14) Henderson, M. A. Surf. Sci. Rep. 2002, 46, 1.

(15) Polunina, I. A.; Isirikyan, A. A.; Polounine, K. E.; Mikhailova, S. S. Colloids Surf., A 1999, 160, 141.

(16) Kimmich, R.; Anoardo, E. Prog. Nucl. Magn. Reson. Spectrosc. 2004, 44, 257.

(17) Ferrante, G.; Sykora, S. Adv. Inorg. Chem. 2005, 57, 405.

(18) De Pasquale, C.; Marsala, V.; Berns, A. E.; Valagussa, M.; Pozzi, A.; Alonzo, G.; Conte, P. J. Soils Sediments 2012, 12, 1211. 
(19) Conte, P.; Marsala, V.; De Pasquale, C.; Bubici, S.; Valagussa, M.; Pozzi, A.; Alonzo, G. GCB Bioenergy 2012, DOI: 10.1111/ gcbb.12009.

(20) Schure, M. R.; Soltys, P. A.; Natusch, D. F. S.; Mauney, T. Environ. Sci. Technol. 1998, 19, 82.

(21) Bakhmutov, V. I. Practical NMR Relaxation for Chemists; Wiley: Chichester, U.K., 2004.

(22) Lauffer, R. B. Chem. Rev. 1987, 87, 901.

(23) Korb, J.-P. Magn. Reson. Imaging 2001, 19, 363.

(24) Korb, J.-P.; Bryant, R. G. Adv. Inorg. Chem. 2005, 57, 293.

(25) Laurent, S.; Forge, D.; Port, M.; Roch, A.; Robic, C.; Elst, L. V.; Muller, R. N. Chem. Rev. 2008, 108, 2064.

(26) Hwang, L.-P.; Freed, J. H. J. Chem. Phys. 1975, 63, 4017.

(27) Gupta, S. M.; Tripathi, M. Chin. Sci. Bull. 2011, 56, 1639

(28) Yurdakal, S.; Palmisano, G.; Loddo, V.; Augugliaro, V.; Palmisano, L. J. Am. Chem. Soc. 2008, 130, 1568.

(29) Beck, D. D.; White, J. M.; Ratcliffe, C. T. J. Phys. Chem. 1986, 90, 3123.

(30) Jug, K.; Nair, N. N.; Bredow, T. Surf. Sci. 2005, 590, 9-20.

(31) Erdogan, R.; Fellah, M. F.; Onal, I. J. Quantum Chem. 2011, 111, 174.

(32) Vittadini, A.; Selloni, A.; Rotzinger, F. P.; Grätzel, M. Phys. Rev. Lett. 1998, 14, 2954. 